\title{
Gas Giant and Ice Giant Atmospheres: Focused Questions for 2023-2032
}

Planetary Decadal Survey White Paper

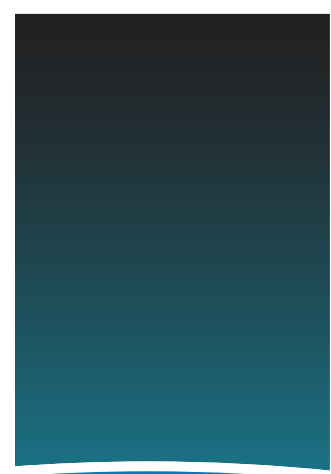

\section{Authors:}

Michael H. Wong (UC Berkeley + SETI Institute)

Statia Luszcz-Cook (Columbia University + AMNH)

Kunio Sayanagi (Hampton Univ.)

Luke Moore (Boston Univ.)

Tommi Koskinen (LPL)

Julianne I. Moses (SSI)

Imke de Pater (UC Berkeley)

\section{Co-signers:}

Shahid Aslam (NASA GSFC)

Sushil K. Atreya (Univ. Michigan)

Kevin H. Baines (JPL + UW-Madison)

Gordon Bjoraker (NASA GSFC)

Katherine R. de Kleer (Caltech)

Scott G. Edgington (JPL)

Jonathan Fortney (UC Santa Cruz)

Thomas K. Greathouse (SwRI)

Heidi B. Hammel (AURA)

Cheng Li (UC Berkeley)

Paul R. Mahaffy (NASA GSFC)

James Sinclair (JPL)

Lawrence A. Sromovsky (UW-Madison)

\section{Contact info:}

mikewong@astro.berkeley.edu

+1 510-224-3411 
We present focused questions in giant planet atmospheres that: encompass many preeminent observational and theoretical results, synergize with terrestrial climate and exoplanet physics/chemistry research, and have clear pathways for progress in the next decade.

\begin{tabular}{|c|c|c|}
\hline Key question & Regions & Path to advancement \\
\hline $\begin{array}{l}\text { Q1. How are planetary formation } \\
\text { and current atmospheric } \\
\text { composition linked? }\end{array}$ & $\begin{array}{l}\text { Deep interior, } \\
\text { troposphere, } \\
\text { stratosphere }\end{array}$ & $\begin{array}{l}\text { Noble gases from probes, gravity field } \\
\text { measurements, IR-radio imaging } \\
\text { spectroscopy, modeling advancements }\end{array}$ \\
\hline $\begin{array}{l}\text { Q2. How does moist convection } \\
\text { shape atmospheric structure in } \\
\text { hydrogen-dominated } \\
\text { atmospheres? }\end{array}$ & Troposphere & $\begin{array}{l}\text { Long-term cadence imaging, } \\
\text { microwave remote sensing, } \\
\text { thermal/composition profiles from } \\
\text { probes, numerical modeling }\end{array}$ \\
\hline $\begin{array}{l}\text { Q3. How do stratospheric } \\
\text { properties trace interactions with } \\
\text { internal and external } \\
\text { phenomena? }\end{array}$ & $\begin{array}{l}\text { Troposphere, } \\
\text { stratosphere }\end{array}$ & $\begin{array}{l}\text { Mid-IR remote sensing, entry probes } \\
\text { for thermal structure, UV spectroscopy }\end{array}$ \\
\hline $\begin{array}{l}\text { Q4. What heating mechanisms } \\
\text { sustain the high temperatures of } \\
\text { the giant planet thermospheres? }\end{array}$ & Thermosphere & $\begin{array}{l}\text { IR and UV spectroscopy, UV and radio } \\
\text { occultations at many latitudes and local } \\
\text { times, GCM modeling }\end{array}$ \\
\hline $\begin{array}{l}\text { Q5. How do external inputs and } \\
\text { local ion chemistry produce the } \\
\text { complex variability observed in } \\
\text { the ionospheres? }\end{array}$ & Ionosphere & $\begin{array}{l}\text { Orbiter mass spectrometry, IR } \\
\text { spectroscopy, radio occultations }\end{array}$ \\
\hline
\end{tabular}

\section{Q1. How are planetary formation and current atmospheric composition linked?}

Bulk planetary abundances and isotope ratios - ultimately constrained by observed atmospheric compositions-constrain properties of the protosolar disk, planet formation processes, and planetary evolution (Atreya et al. 2020). For example, formation by core accretion predicts enrichments in heavy elements relative to solar values (e.g., Helled et al., 2014). Methane abundances from occultations and visible/infrared spectra reveal that all four giant planets are enriched in carbon relative to solar, with higher enrichments for the outermost planets (Fig. 1). Spectroscopic challenges and sequestration in deep clouds have rendered other abundances more difficult to measure. At Jupiter, the Galileo probe measured the composition down to pressures of 15-20 bar, finding solar noble gas isotopic ratios (Mahaffy et al. 2000). The noble gases and volatile species $\mathrm{CH}_{4}, \mathrm{NH}_{3}$, and $\mathrm{H}_{2} \mathrm{~S}$ were all enriched by a factor of $\sim 4$ (Wong et al. 2004) relative to the protosolar value (Asplund et al. 2009). Meteorology associated with the probe descent into a 5- $\mu \mathrm{m}$ hotspot (Showman and Ingersoll 1998) foiled attempts to measure the atmospheric $\mathrm{O} / \mathrm{H}$ ratio, and other measurements have found it to be solar or supersolar (e.g., Wong et al. 2008, Bjoraker et al. 2018, Li et al. 2020). For the other three giant planets, in situ probe measurements do not yet exist. Early disk-integrated radio observations of Uranus and Neptune (Gulkis et al. 
1978, de Pater et al. 1991) and recent high-resolution maps with the VLA and ALMA (Molter et al. 2020, Tollefson et al. 2020) reveal that the N/S ratios of Uranus and Neptune are well below Jupiter's roughly solar N/S ratio. This finding is further supported by infrared detections of $\mathrm{H}_{2} \mathrm{~S}$ in the upper atmospheres of both ice giants (Irwin et al. 2018,2019), and could point to chemistry in deep clouds (Atreya et al. 2019) or a formation scenario involving trapping of $\mathrm{NH}_{3}$ and $\mathrm{H}_{2} \mathrm{~S}$ into clathrates within planetesimals (Hersant et al. 2004). Deep composition may also be constrained by measuring disequilibrium species-molecules like $\mathrm{CO}$ that in equilibrium are confined to the warm planetary interiors (e.g., Fegley and Lodders, 1994). Measurements of tropospheric CO (e.g., Lellouch et al., 2005, Luszcz-Cook and de Pater, 2013) place constraints on deep O/H abundances, but these constraints are dependent on thermochemical and kinetic models (Cavalié et al. 2017, Venot et al. 2020).
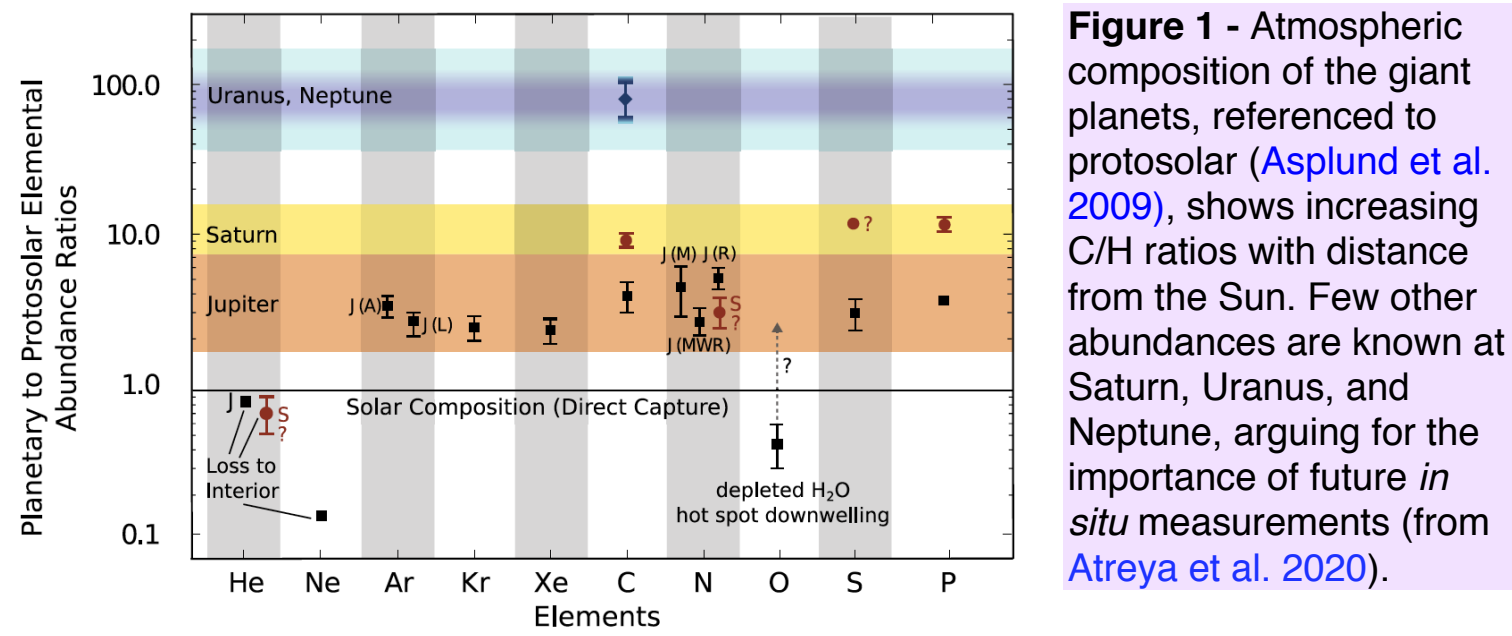

Atmospheric composition is linked to bulk planetary composition via interior models. At Jupiter, older compact core models are challenged by Juno's high-precision gravitational field measurements, which suggest a fuzzy, dilute core (Wahl et al. 2017). A dilute core depends on processes including layered convection (Müller et al. 2020, Leconte and Chabrier 2012), core erosion (Guillot et al. 2004, Moll et al. 2017), a giant impact, or significant migration in Jupiter's history.

Comparative planetology and broader scope: Comparisons of composition and structure across the giant planets places constraints on formation conditions, such as gradients in the early solar nebula, and on subsequent evolution. Of particular interest are the ice giants, which are similar in mass but show striking differences in average density and internal heat flow. The solar system giant planets provide the benchmark for connecting observations of exoplanet density and spectroscopy with giant exoplanet composition, and thus with exoplanet formation.

New data: In situ mass spectral measurements are required for volatile and noble gas abundances and isotope ratios on Saturn, Uranus, and Neptune. Higher resolution maps of multiple disequilibrium species and accurate vertical profiles of $\mathrm{CO}$ (in situ or from ALMA/ngVLA) constrain deep composition. Gravity data for Uranus and Neptune would provide necessary constraints on interior structure to better connect the atmosphere, bulk composition, and formation history. Advances in modeling and laboratory measurements are needed to constrain accretion during formation, interior structure and evolution, and cloud chemistry. 


\section{Q2. How does moist convection shape atmospheric structure in hydrogen- dominated atmospheres?}

The tropospheres of giant planets are heated from below, with the amount of heat radiated to space exceeding the amount absorbed from the Sun (except possibly for Uranus; Li et al. 2010, 2012, Pearl et al 1990, Pearl and Conrath 1991). Convection is largely responsible for the vertical heat transport in the tropospheres of the giant planets, but the process is fundamentally different from the terrestrial case, because moist air is heavier than dry air in hydrogen-dominated atmospheres. Molecular weight stratification provides a stabilizing influence against convection, with solar or subsolar enrichments of volatiles enabling continuous weak convection, and higher enrichments leading to episodic convective outbreaks (Li and Ingersoll 2015, Sugiyama et al. 2014). The apparent lack of an internal heat source at Uranus may be an effect of observing the planet in between convective outbreaks (Smith and Gierash 1995), or even a permanent barrier to convection (Friedson and Gonzales 2017). Seasonal forcing may affect Saturn's convective outbreaks (Sánchez-Lavega et al 2020).

Moist convection and meridional overturning circulation are linked. One marker of active convection is lightning. On Jupiter, lightning frequency is much higher in cyclonic zonal wind bands (Little et al. 1999), which are traditionally associated with dark belts and considered to be the downwelling branches of meridional cells (Hess and Panofsky 1951). To explain the apparent inconsistency between downwelling and convective activity Jupiter, vertically stacked meridional circulation cells have been proposed (Ingersoll et al. 2000, Showman and de Pater 2005). However, Juno's observations of deep $\mathrm{NH}_{3}$ depletions at Jupiter do not provide obvious support for the stacked-cell scenario (Bolton et al. 2017, Li et al. 2017). The volatiles $\mathrm{CH}_{4}$ and $\mathrm{H}_{2} \mathrm{~S}$ in the ice giants also show latitudinal gradients and banding, and again stacked cells have been proposed, but consensus has not been reached on their configurations (Fig. 2, Sromovsky et al. 2014, Molter et al. 2020, Fletcher et al. 2020). Mysteriously, the ice giant compositional bands have no counterparts in the known zonal wind field. Moist convection may also play an important role in shaping the thermal structure of vortices, which differs from their surroundings (Wong et al 2011). Moist convection drives the formation of tropospheric and stratospheric vortices on Saturn (Sayanagi et al. 2013, Fletcher et al. 2012), and possibly large-scale structures such as zonal jets and polar vortices (Showman 2007, Sayanagi et al. 2008, Brueshaber et al. 2019).
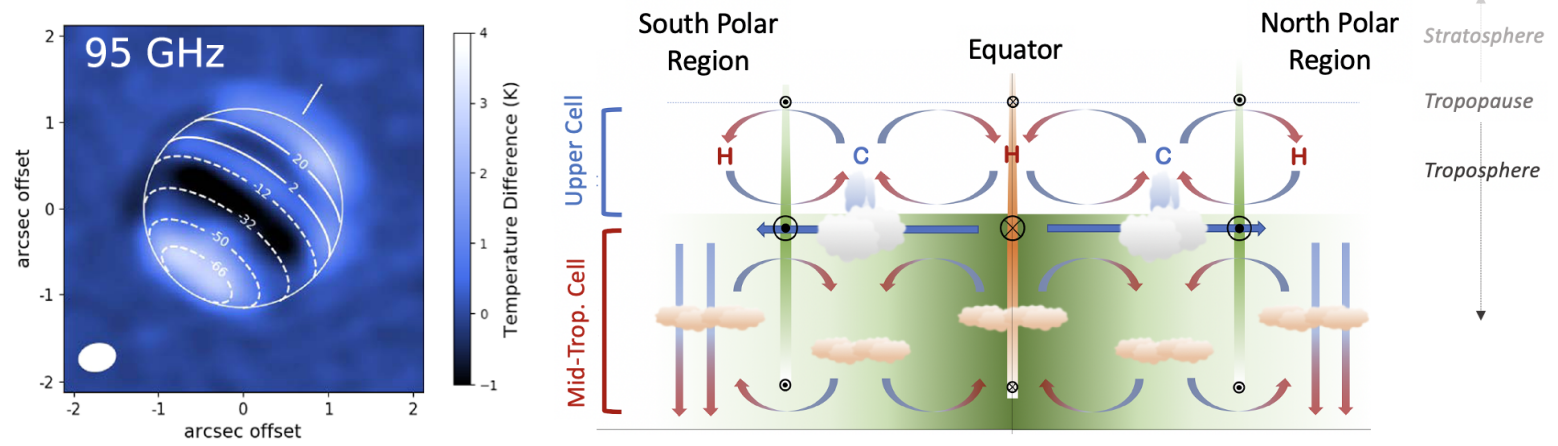

Figure 2 - Left: Millimeter maps show banded structure indicative of variation in $\mathrm{CH}_{4}$ and $\mathrm{H}_{2} \mathrm{~S}$ concentrations (Tollefson et al. 2019), offering new capability to trace upwelling/ downwelling circulation on Neptune. Right: One of the meridional circulation schemes proposed by Sromovsky et al. (2014) for Uranus and Neptune (adapted from Fletcher et al. 2020). 
Comparative planetology and broader scope: The abundance of volatile species controls the style of moist convection in giant planet atmospheres (Leconte et al. 2017), but quantitative tests of this effect require comparing activity in the four giant planets and even the Earth. Understanding convective heat transport is essential for constraining the thermal evolution of our giant planets, helping to distinguish between thermal evolution models used to interpret exoplanet observations (particularly directly-imaged exoplanets; Fortney and Nettelmann 2010, Spiegel et al. 2012).

New data: Probe measurements at Saturn, Uranus, and Neptune, akin to the Galileo probe at Jupiter, would be of immense value for constraining composition and thermal structure below the clouds. Microwave radiometry data, like that provided for Jupiter by Juno, and remote microwave observations will reveal deep gradients and spatial variations (Fig. 2); the ngVLA would greatly expand ground-based capabilities in this area (de Pater et al. 2020). Given spatial variability, in situ results would be greatly enhanced by multiple probes, perhaps accompanied by miniaturized secondary probes with limited payloads (Sayanagi et al. 2020); ice giant orbiters would need spectral resolution in the visible and IR for vertical profiles of composition and aerosols. Cadence observations (e.g., Simon et al. 2015) are needed to measure evolution on timescales of individual convective events to seasonal cycles. Continued numerical model development is needed to advance understanding of individual processes and fluid dynamics under realistic assumptions.

\section{Q3. How do stratospheric properties trace interactions with internal and external phenomena?}

Remote observations of the stratosphere - the radiative region just above the troposphere - reveal fascinating variability in composition and temperature that traces diverse interactions with both external and internal phenomena. Solar radiation heats the stratosphere and initiates photolysis of methane, creating a slew of hydrocarbon chemical products and associated hydrocarbon haze layers. Seasonal changes in forcing are expected to drive spatial and temporal variations in chemistry, although the precise nature of the seasonal effect is poorly understood. For example, Uranus exhibits mid-infrared brightness variations that do not match seasonal photochemical model predictions (Moses et al. 2018). Material entering the atmosphere from the environment also contributes to the chemistry. Oxygen-bearing species have been detected on all four giant planets (Feuchtgruber et al. 1997, Lellouch et al. 1997), indicating infall from rings, icy satellites, interplanetary dust, and/or comets. As demonstrated by the 1994 impact of Comet ShoemakerLevy 9 with Jupiter, comet impacts can have a dramatic, spatially and temporally variable influence on the atmospheric composition and chemistry (Lellouch et al. 1995, Noll et al. 1995). Detections of CO, HCN, and CS in Neptune's stratosphere indicate significant cometary impact(s) in the past centuries (Lellouch et al. 2005, Luszcz-Cook and de Pater 2013, Moreno et al. 2017).

Stratospheric temperature oscillations and anomalies trace interactions with tropospheric processes: gravity waves propagate upward, interact with jets and deposit energy through wave breaking. Fast low-latitude stratospheric jets exhibit periodic temperature variability on Jupiter and Saturn, thought to be driven by gravity wave interactions analogous to the quasi-biennial oscillation on Earth (Leovy et al. 1991, Orton et al. 2008). The "beacon," an unanticipated $80 \mathrm{~K}$ warming in Saturn's stratosphere in response to the 2010 Great Storm, was likely established by gravity wave interactions with stratospheric jets (Fletcher et al. 2012; Fig. 3). This process at Saturn might help to interpret stratospheric hot spots on Neptune, where turbulent energy sources may lie too deep for direct observation (Hammel et al. 2007, Orton et al. 2007). 


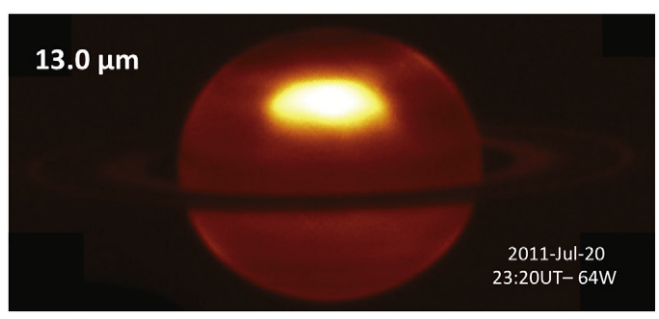

Figure 3 - Energy input for Saturn's "beacon" came from interactions between gravity waves propagating from the turbulent disturbance and the background stratospheric flow (Fletcher et al. 2012).

Comparative planetology and broader scope: The range of obliquities and orbital periods of the giant planets covers different amplitudes and timescales of seasonal forcings. Data on giant planet impacts are important for constraining impactor size distributions (Hueso et al. 2013), which also relate to outer solar system cratering rates and surface ages. Stratospheric oscillations similar to those on Jupiter and Saturn are actively studied at Earth, and potentially on exoplanets (Léard et al. 2020, Barton and McCormack 2017, Showman et al. 2019).

New data: UV and mid-IR spectroscopy (e.g., from JWST) can yield temperature and compositional profiles, while imaging reveals gradients and discrete features. Long-duration cadence observations are needed to resolve periodic oscillations with timescales of multiple years, and disruptions in these oscillations. Degeneracies in occultation inversions can be resolved by in situ probe entry data, and net flux radiometry can characterize radiative balance.

\section{Q4. What heating mechanisms sustain the high temperatures of the giant planet thermospheres?}

Above the homopause level, radiative cooling in giant planet atmospheres rapidly decreases as the $\mathrm{CH}_{4}$ abundance drops. But the reduced cooling efficiency is not sufficient to explain the unexpectedly high temperatures observed at low latitudes, leading to the "energy crisis" problem in giant planet thermospheres (Fig. 4; García Muñoz et al. 2017). Energy sources include the breaking of gravity waves propagating upward, and resistive heating resulting from auroral currents. Wave propagation can be challenging to treat accurately in GCMs, which have not been able to capture the necessary meridional transport of auroral heating at the poles. Cassini INMS data provides evidence of wave damping in Saturn's thermosphere (Müller-Wodarg et al. 2019), implying that Rayleigh drag can facilitate equatorward heat transport against the "Coriolis barrier" on rapidly rotating planets (Smith et al. 2007).

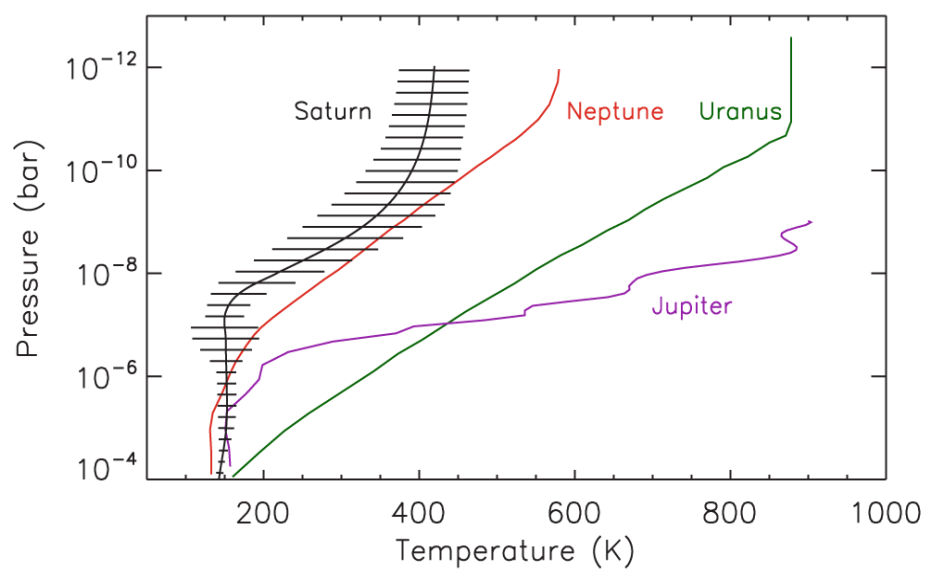

Figure 4 - Low-latitude thermospheric temperatures increase with altitude as the decreasing abundance of $\mathrm{CH}_{4}$ inhibits radiative cooling. But the differences among the planets are not explained by models, which predict temperatures of only 130-200 $\mathrm{K}$ at low latitudes (Yelle and Miller 2004). Figure from García Muñoz et al. (2017).

Comparative planetology and broader scope: Thermospheric temperature excesses across the giant planets are not correlated with insolation or even with auroral power. Although modeling 
advances are needed to demonstrate understanding of the thermospheric heat budgets, solutions can only be validated if they can explain temperature profiles in all four giant planets. The diversity of magnetic field configurations among the planets provides a test of relevant physical processes in models, and including these processes may lead to new insights into seasonal climate cycles in other layers such as stratospheres and ionospheres.

New data: Ice giant orbiters with appropriate trajectories and instrumentation would be able to determine neutral densities and find evidence for wave damping, as Cassini INMS did at Saturn. Temporal and spatial variability can be measured by UV occultations over a range of latitudes and local times, providing the main constraints for heating and circulation. UV spectroscopy of auroral emissions are crucial for establishing composition, temperatures, and energy states of thermospheric species.

\section{Q5. How do external inputs and local ion chemistry produce the complex variability observed in the ionospheres?}

Cassini's long tour in the Saturn system has revealed ionospheric variability that has not yet been satisfactorily explained and has uncovered a complicated and unforeseen coupling between Saturn's atmosphere and rings. During Cassini's Grand Finale, two populations of infalling ring particles were discovered: a massive flux of primarily neutral nanograins peaked at the equator (Perry et al. 2018), and a flux of charged grains spiraling in along magnetic field lines at midlatitudes, a process termed "ring rain" (Hsu et al. 2018). These ring particles appear to cause the observed non-solar structures in $\mathrm{H}_{3}{ }^{+}$emission and low-latitude depletion of electron density, but explanations for vertical and temporal electron density variations remain unclear: they might be related to rapid flux variations of infalling ring material, to gravity waves breaking in the lower thermosphere, and/or to some other extreme ionization or transport process.

Uranus also demonstrates upper atmosphere variability: thermospheric temperatures, derived from $\mathrm{H}_{3}{ }^{+}$observations, vary on timescales exceeding the length of a solar cycle; the variations do not appear to correlate with season (Melin et al., 2011). However, seasonal change and spatial and temporal variability in $\mathrm{H}_{3}{ }^{+}$abundances could be influencing derivations of temperature (Moore et al., 2019). For Neptune, $\mathrm{H}_{3}{ }^{+}$has not yet been detected, and models of the ionosphere predict $\mathrm{H}_{3}{ }^{+}$ concentrations greater than the current upper limit (Melin et al. 2018). This may suggest that the thermospheric temperature and/or methane homopause level differ from what was observed by Voyager, or that an influx of external material, perhaps from Triton/Neptune's rings, has depleted ionospheric $\mathrm{H}_{3}{ }^{+}$densities. While many other ionic species are predicted to be present on the ice giants, none have yet been detected (Moses et al. 2020).

Comparative planetology and broader scope: Although overall composition should be similar in the ionospheres of the giant planets, the insolation, seasonal forcing, magnetic field configuration, and ring influxes are very different.

New data: Ice giant orbital tours should provide good latitudinal coverage by radio occultations to constrain ionospheric variability. Ion composition from mass spectral measurements during "deep dive" orbiter passes can validate model predictions for major species like $\mathrm{H}_{3}{ }^{+}$and $\mathrm{H}^{+}$, and measure external influxes from rings, magnetospheric plasma, and micrometeorites. Limb scans by high-resolution IR and UV spectrometers can also provide much-needed insight into vertical ionospheric and thermal structure. 


\section{Summary}

The focused questions presented here argue strongly for a multi-pronged and collaborative research strategy in the coming decade. Orbiters (with probes) must be paired with long-term remote imaging and spectroscopy campaigns across multiple wavelengths and advances in models of planet formation, evolution, circulation, and chemistry. Funding for individual investigator programs will serve as a critical complement to large-scale mission support.

\section{References}

Asplund, M. et al. (2009) ARA\&A 47, 418. Atreya, S.K. et al. (2020) SSRv 216, 18. Atreya, S.K., et al. (2019) Acta Astron 162, 266. Barton, C.A. \& J.P. McCormack (2017) GeoRL 44, 150.

Bjoraker, G.L. et al. (2018) AJ 156, 101.

Bolton, S.J., et al. (2017) Sci 356, 821.

Brueshaber, S.R., et al. (2019) Icar 323, 46.

Cavalié, T. et al. (2017), Icar 291, 1.

de Pater, I. et al. (2020) Decadal white paper, this issue. de Pater, I., et al. (1991) Icar 91, 220.

Fegley, Jr.B. and K. Lodders (1994), Icar 110, 117. Feuchtgruber, H. et al. (1997) Nature 389, 159.

Fletcher, L.N., et al. (2020) SSRv 216, 21.

Fletcher, L.N. et al. (2012) Icar 221, 560.

Folkner, W.M. et al. (1998) JGR 103, 22847.

Fortney, J.J. \& N. Nettelmann (2010) SSRv 152, 423.

Friedson, A.J. \& E.J. Gonzales (2017) Icar 297, 160.

García Muñoz, A. et al. (2017) in Handbook of Exoplanets, Springer, Cham, p.1.

Guillot, T. et al. (2004) in Jupiter, Cambridge, p.53.

Gulkis, S. et al. (1978) Icar 34, 10.

Hammel, H.B. et al. (2007) AJ 134, 637.

Helled, R.S. et al. (2014) Protostars \& Planets VI, 643. Hersant, F. et al. (2004) P\&SS 177, 104680.

Hess, S.L. \& A.A. Panofsky (1951) in Compendium of Meteorology, AMS, Boston, p.391.

Hsu, H.-W., et al. (2018) Sci 362, aat3185.

Hueso, R. et al. (2013) A\&A 560, A55.

Ingersoll, A.P. et al. (2000) Natur 403, 630.

Irwin, P.G.J. et al. (2018) Nat Astron 2, 420.

Irwin, P.G.J. et al. (2019) Icar 329, 550.

Léard, P. et al. (2020) PhRvF 5, 024801.

Leconte, J. et al. (2017) A\&A 598, A98.

Leconte, J. and G. Chabrier. (2012) A\&A 540, A20.

Lellouch, E. et al. (2005) A\&A 430, L37.

Lellouch, E. et al. (1997) PlanSS 45, 1203.

Lellouch, E. et al. (1995) Nature 373, 592.

Leovy, C.B. et al. (1991) Nature 354, 6352.

Li, C. et al. (2020) Nat Astron 4, 609.

Li, C. \& A.P. Ingersoll (2015) NatGe 8, 398.

Li, C. et al. (2017) GRL 44, 5317.

Li, L. et al. (2010) JGRE 115, E11002.

Li, L. et al. (2012) JGRE 117, E11002.
Little, B. et al. (1999) Icar 142, 306.

Luszcz-Cook, S. \&I. de Pater (2013) Icar 222, 379.

Mahaffy, P.R. et al. (2000) JGR, 105, 15061.

Melin, H. et al. (2018) MNRAS 474, 3714.

Melin, H. et al. (2011) ApJ 729, 134.

Moll et al. (2017). ApJ 849, 24.

Molter, E. M. et al. (2020) PSJ, submitted.

Moore, L. et al. (2019) RSPTA 377, 20190067.

Moreno, R. et al. (2017). A\&A 608, L5.

Moses, J.I. et al. (2020) RSPTA, submitted.

Moses, J.I. et al. (2018) Icar 307, 124.

Müller, S., R. et al. (2020) A\&A 638, A121.

Müller-Wodarg, I.C.F. et al. (2019) GeoRL 46, 2372.

Noll, K.S. et al. (1995) Science 267, 1307.

Orton, G.S. et al. (2008) Nature 453, 196.

Orton, G.S. et al. (2007) A\&A 473, L5.

Pearl, J.C. \& B.J. Conrath (1991) JGR 96, 18921.

Pearl, J. C., et al. (1990) Icar 84, 12.

Perry, M.E., et al. (2018) GeoRL 45, 10,093.

Sánchez-Lavega, A., et al. (2020) NatAs 4, 180.

Sayanagi, K.M. et al. (2020) SSRv 216, 72.

Sayanagi, K.M. et al. (2013) Icar 223, 460.

Sayanagi, K.M., A.P. Showman, \& T.E. Dowling (2008) JAtS 65, 3947.

Showman, A.P. et al. (2019) ApJ 883, 4.

Showman, A.P. (2007) JAtS 64, 3132.

Showman, A.P. \& I. de Pater (2005) Icar 174, 192.

Showman, A.P. \& A.P. Ingersoll (1998) Icar 132, 205.

Simon, A.A., M.H. Wong, \& G.S. Orton (2015) ApJ 812, 55.

Smith, C.G.A., et al. (2007) Natur 445, 399.

Smith, M.D. \& P.J. Gierasch (1995) Icar 116, 159.

Spiegel, D.S. \& A. Burrows (2012) ApJ 745, 174.

Sromovsky, L.A. et al. (2014) Icar 238, 137.

Sugiyama, K. et al. (2014) Icar 229, 71.

Tollefson, J. et al. (2020). PSJ, submitted.

Tollefson, J. et al. (2019) AJ 157, 251.

Venot, O., et al. (2020) A\&A 634, A78.

Wahl, S.M. et al. (2017) GeoRL 44, 10.

Wong, M.H. et al. (2011) Icar 215, 211.

Wong, M.H. et al. (2008) RiMG 68, 219.

Wong, M.H. et al. (2004) Icar 171, 153.

Yelle, R.V. \& S. Miller (2004) in Jupiter, Cambridge, p. 210 . 